\title{
Emerging viral diseases from a vaccinology perspective: preparing for the next pandemic
}

\author{
Barney S. Graham ${ }^{\star \star}$ and Nancy J. Sullivan`
}

\begin{abstract}
Emerging infectious diseases will continue to threaten public health and are sustained by global commerce, travel and disruption of ecological systems. Most pandemic threats are caused by viruses from either zoonotic sources or vector-borne sources. Developing better ways to anticipate and manage the ongoing microbial challenge will be critical for achieving the United Nations Sustainable Development Goals and, conversely, each such goal will affect the ability to control infectious diseases. Here we discuss how technology can be applied effectively to better prepare for and respond to new viral diseases with a focus on new paradigms for vaccine development.
\end{abstract}

$$
\text { r }
$$

nfectious diseases pose the greatest threat to public health and result in more years of life lost from premature death than any other disease process ${ }^{1,2}$. Prior to the 19th century, infection syndromes were often described better by artists and writers than by scientists. This began to change as microbes were better understood, and for the past 150 years, many ancient diseases such as smallpox, polio and measles have been explained and controlled. However, over the past three decades, there has been a constant stream of newly identified pathogens that have received increasing attention. The approach to these new pandemic threats has generally been reactive, and specific medical interventions have not been available in time to make a substantial impact on the immediate outbreak. Technical advances have provided tools that have made a more proactive approach feasible and a critical determinant for achieving the Sustainable Development Goals (SDGs) established by the UN (United Nations) in 2015 (Table 1). The introduction of emerging infectious diseases (EIDs) into human populations is often a consequence of economic development that brings zoonotic reservoirs in closer proximity to people, and the spread of EIDs has contributed to a number of inter-related factors that affect global human wellness. Notably, the control of infectious disease is interdependent with progress toward the $17 \mathrm{UN} \mathrm{SDGs}^{3}$. Therefore, prevention of and preparedness for epidemics should be conducted in the context of meeting SDGs.

The accelerated appearance of new infectious disease threats with pandemic potential is reflected by the establishment of the WHO (World Health Organization) Emergency Committee in 2005. Since then, four Public Health Emergencies of International Concern have been declared. These involved the pandemic H1N1 influenza in 2009, the polio resurgence in 2014, the Ebola epidemic in West Africa in 2014 and the emergence of Zika virus in the Americas in 2016. Whether the frequency has been amplified by increasing global commerce and travel, disruption of ecologies from conflict or economic development that brings humans into contact with previously unrecognized pathogens, humans are faced with perpetual microbial challenge and an increasing occurrence of pandemic threats to global health ${ }^{4}$.

Most EIDs are caused by viruses that are either zoonotic or vector-borne. While antimicrobial resistance is also of considerable concern, the prospects for pandemic spread of a bacterial or fungal pathogen seem less likely. Nonetheless, the spread of diseases such as plague, cholera, tuberculosis and, more recently, Legionnaire's disease, invasive streptococcal disease and toxic-shock syndrome would present considerable risk. Thus, there is a need for rapid diagnosis, definition of transmission pathways and availability of antimicrobial agents. Here we will focus on newly emerging viral diseases, particularly those identified over the past three decades, during which the mobility of humans, transmission vectors and viruses has been on a steep upward trajectory, and consider the immunological tools and new technologies that could help address this problem. Improving scientific preparedness for the next pandemic threat can be divided into four broad categories, which we will consider in turn below.

\section{Category 1: pathogen surveillance and discovery}

The establishment of centralized infrastructure for high-throughput sequencing in regions with high biological diversity in which new human infections are most likely to occur will allow the rapid detection of emerging pathogens. These centers could also serve as hubs from which to deploy resources to unexpected locations in response to new outbreaks. The same infrastructure and technologies could be used to probe the environment on an ongoing basis to define the spectrum of viral strains and species that exist within phylogenetic families known to infect humans and to thoroughly define all existing viruses via high-throughput sequencing. Improved capacity for surveillance can be leveraged to improve and develop diagnostics because of technology convergence, and justifies the investment in facilities, personnel and training. The combination of improved surveillance and diagnostics will further enhance regional patient care and sustainable technical competence.

\section{Category 2: basic biology and development of reagents and assays}

For prototypic viruses within each of the main families of viruses that cause human infection, there is a need to develop a morecomprehensive set of reagents, including monoclonal antibodies that recognize both surface proteins and internal proteins. This would allow the development of antigen-specific assays and provide standards for the early development of serological assays. This also involves defining the key antigenic targets for protective antibodies, 


\section{Table 1 | Interface between SDGs and the risk of emerging infectious diseases}

Goals for the control of infectious disease

Reduce human contact with pathogens found in conditions of poor sanitation (rodent- and vector-borne diseases), alternative food sources (bushmeat hunting), untreated water (parasites and bacteria) and altered-pathogen reservoirs resulting from climate change or deforestation.

Reduce pathogen exposure and disease severity via better understanding of how infectious diseases are transmitted, lowering resistance to seeking care and knowing the value of medical interventions such as vaccination.

Reduce the spread of sexually transmitted viruses, such as HIV and HPV, for which young women have the highest risk of acquisition.

Reduce exposure to mosquitoes and other transmission vectors by improving and maintaining general infrastructure and living conditions (reduce standing water, protect indoor spaces with screens); build capacity for surveillance and early diagnosis in low- and middle-income countries and maintain public health systems and access to medical care to contain outbreaks and prevent pandemics.

Reduce pathogen transmission from high-risk occupations related to the hunting or selling of wild animals in mixed-species marketplaces and diminish the prevalence of commercial sex work and crowded living conditions that provide avenues for the transmission of some viruses.
Relevant SDG(s)

1 , No poverty; 2, Zero hunger; 6 , Clean water and sanitation; 13, Climate action; 14, Life below water; 15, Life on land

3, Good health and well-being; 4, Quality education

5 , Gender equality

7, Affordable and clean energy; 9, Industry, innovation and infrastructure; 10 , Reduced inequalities; 11 , Sustainable cities and communities; 12 , Responsible consumption and production; 16 , Peace, justice and strong institutions; 17 , Partnership for the goals

8, Decent work and economic growth

The goals detailed at left are related to specific goals (right) among the 17 UN SDGs ${ }^{3}$. HPV, human papillomavirus.

solving the structures of surface glycoproteins, developing serological assays to measure functional activity with either recombinant or pseudotyped reporter viruses and, where feasible, identifying cellular receptors and tropism. This basic information would provide the toolbox needed for defining mechanisms of neutralization and developing animal models of pathogenesis. Determining whether replication is restricted by entry receptors (for example, betacoronaviruses), innate cellular factors (for example, APOBEC3G for human immunodeficiency virus type 1 (HIV-1)) or failure to inhibit interferon (for example, flaviviruses or paramyxoviruses) or whether viruses have unrestricted replication (for example, filoviruses) or can be easily adapted and selected (for example, influenza virus) for growth in mice would enable preclinical studies for early product development.

\section{Category 3: vaccine design and preclinical development}

On the basis of viral entry mechanisms, tropism, pathogenesis and epidemiological considerations, vaccine antigen designs and selected delivery approaches could be initiated for prototypic viruses. As suggested below, this type of pre-existing information for flaviviruses substantially accelerated timelines for the development of a vaccine against Zika virus. Immunologists and virologists will be essential for the refinement of approaches for testing the authenticity of antigenic surfaces. For example, defining antibody lineages associated with broad neutralizing activity, as has been done for $\mathrm{HIV}^{5-7}$ and influenza virus ${ }^{8}$, can guide the development of epitopespecific germline versions of - B cell antigen receptor-expressing Raji cells that can serve as a tool for screening antigen designs? This has facilitated the development of novel antigens for HIV ${ }^{10}$ and influenza virus ${ }^{11}$. While many of the rules that govern the immunogenicity of vaccine antigens displaying isolated epitopes are yet to be defined, having antibodies with the desired functional properties to confirm the preservation of targeted epitopes in vaccine antigens is a critical part of rational design ${ }^{12,13}$. Genes encoding the relevant human antibodies can also be built into mice for in vivo assessment of antigenicity ${ }^{14}$. Assays to define the fundamental mechanisms of immunity for prototypic pathogens will guide the approach for vaccine antigen delivery and will directly inform the types of assays needed to define correlates of protection. Those will probably be distinct from the assays needed for surveillance and diagnosis. Likewise, animal-model development is essential, but it is important to distinguish the adequacy of model development for basic research from the adequacy of models needed to support regulatory filings for product licensure. For example, models for studying susceptibility to infection, tropism, pathogenesis and basic mechanisms of immunity will probably use challenge routes and doses different from those used by models attempting to faithfully replicate the infectious exposure that occurs in humans. Such refinements will facilitate accurate bridging of protective immune responses to human immunogenicity data needed to satisfy the requirements for licensure based on animal efficacy (for example, US Food \& Drug Administration (FDA) Animal Rule 21 CFR 601.90).

\section{Category 4: manufacturing and clinical evaluation}

Methods and infrastructure for rapid manufacturing and clinical evaluation of candidate vaccines will be needed, preferably before a pathogen emerges. This will be facilitated by the development of platform technologies for antigen delivery. Assays for monitoring product potency during manufacturing may differ substantially from preclinical research assays. In an outbreak setting in which high incidence rates might quickly wane, having defined immunological correlates of protection will determine the laboratory endpoints used for clinical evaluation of vaccine candidates and will facilitate the authorization of new products for outbreak control. Regional pre-approved protocols for testing efficacy in outbreak settings also improve the chances of meeting product-licensure criteria.

\section{Organizational opportunities}

Much of the scientific knowledge base and infrastructure, particularly as it relates to infectious diseases, has been built in response to crisis. The HIV-1 epidemic began as a zoonotic transmission that emerged and circulated in human populations for decades before exploding into a pandemic disease fueled by human mobility and poverty ${ }^{15}$. The enormous scientific effort to understand the pathogenesis of HIV, as well as work to develop antiviral agents and vaccines, has been a major contributor to the evolution of modern immunology. The HIV pandemic also illustrated the disruptive effect of infectious diseases on economies and social stability, clarified the importance of taking a more global view toward clinical research and directly led to the development of a substantial worldwide clinical-trials infrastructure. The occurrence of severe acute respiratory syndrome (SARS) coronavirus in 2003 (refs ${ }^{16,17}$ ) and 

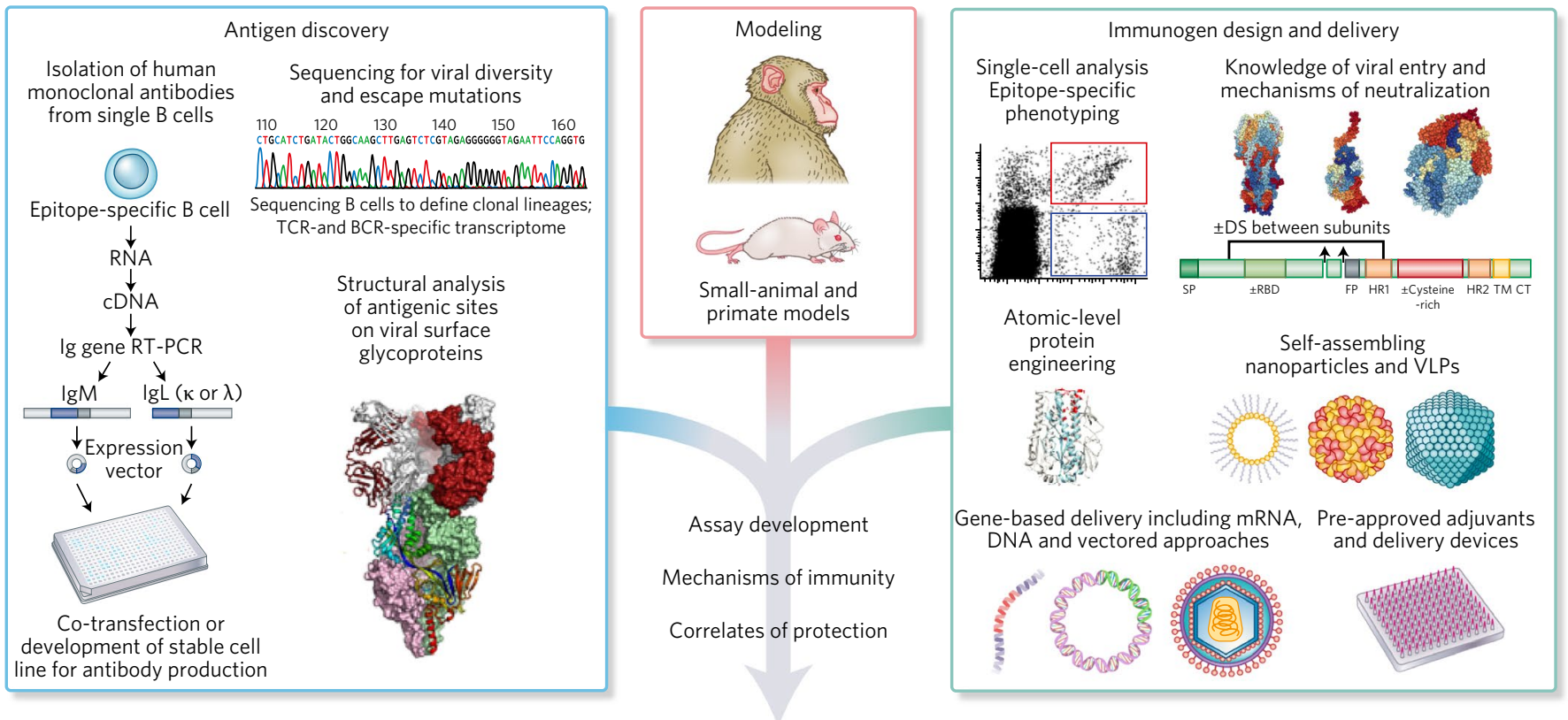

Candidate vaccine

Fig. 1 | Emerging technologies support a new paradigm for vaccine development. Several new or improved technologies over the past 10 years have provided the tools needed for rational vaccine design. They have also created opportunities for more-rapid vaccine development. Structure-guided antigen design is a central feature of this new paradigm. Atomic-level detail of antigenic surfaces, the ability to identify monoclonal antibodies via the cloning of immunoglobulin-encoding genes from specifically sorted B cells, and high-throughput sequencing technology have provided the basis for selecting antigen targets for vaccine-induced immune responses to initiate the design cycle. CRISPR-Cas9-like targeted gene editing has made it possible for animal models to be established on the basis of knowledge of receptor requirements for viral entry and restriction factors that might be species specific. Analysis of immune responses by flow cytometry to define the phenotype of individual cells on the basis of protein- or gene-expression patterns can provide information on repertoire and temporal patterns of the immune response for bridging endpoints to human infection or vaccination. Knowledge of the structure, function and epitope locations for class I fusion proteins across families of viruses provides a basis for selecting these as vaccine targets and for initial antigen designs. Having functional monoclonal antibodies to test antigens for authentic binding surfaces can guide the protein engineering needed to make immunogens. Recognition by B cells is facilitated when antigens are displayed in ordered arrays, and self-assembling nanoparticles provide a vehicle for presenting vaccine antigens in this way. The advent of gene-based expression of antigens from nucleic acids or vectors, advances in adjuvant formulations, and microneedle patches (bottom right) or needle-free or alternative inoculation devices can also contribute to the shortening of timelines and improved efficacy of new vaccines. Ig, immunoglobulin; $\kappa$ and $\lambda$, components of the immunoglobulin light chain (IgL); TCR, T cell antigen receptor; $B C R, B$ cell antigen receptor; $\pm D S$, with or without disulfide bonds; $S P$, signal peptide; $\pm R B D$, with or without a receptor-binding domain; FP, fusion peptide (upward arrowheads indicate upstream cleavage sites); HR1 or HR2, heptad repeat 1 or 2; TM, transmembrane region; CT, carboxyl terminus. Credit: Debbie Maizels/Springer Nature.

the threat of avian influenza virus that emerged in 2005 (refs. ${ }^{18,19}$ ) resulted in the development of a more-robust laboratory surveillance network than was previously available. The rapid succession of the West African Ebola epidemic and the Zika epidemic in South and Central America and the Caribbean galvanized organizations concerned about EIDs and focused the attention of the media as never before. There might now be the political will to support a more-organized global approach to advanced preparation for the next pandemic threat, and much of this could be accomplished within the context of sustainable development.

Ideas for funding and accomplishing more-rapid advanced development of biologicals for outbreak intervention have also evolved out of necessity in response to more-frequent public-health emergencies. For example, within the US Department of Health and Human Services, the office of the Assistant Secretary for Preparedness and Response was established in 2007; under the auspices of this office, the Biomedical Advanced Research and Development Authority can rapidly purchase and support the development of medical countermeasures for pandemic threats. The WHO established a Department of Pandemic and Epidemic Diseases in 2011 to better prepare for and respond to EIDs. New organizations have proposed funding for advanced preparation of vaccine candidates before future and predictable infectious disease outbreaks of global concern occur.
The Coalition for Epidemic Preparedness Innovations was motivated largely by the 2014 Ebola epidemic in West Africa. It proposes to establish a system to fund the development of vaccines in advance of the next epidemic and to prepare products and processes that allow rapid deployment and testing in an outbreak setting ${ }^{20,21}$. Other proposals for biodefense preparedness organizations call for advanced funding of companies with established platform technologies that could be used to develop vaccines against pathogens with pandemic potential, define scalable commercial processes, evaluate safety and immunogenicity through phase II and stockpile a substantial number of doses for efficacy testing in the event of future outbreaks ${ }^{22}$. Therefore, it is reasonable to ask what new technologies are available to address emerging viral diseases in a more proactive way and what can be learned from recent outbreaks that will inform preparations for future infectious disease threats.

\section{Biological and technical opportunities}

In the setting of a new pandemic threat, without the advantage of time and prior knowledge of viral pathogenesis and experience with viral growth and attenuating determinants, it is likely that traditional live-attenuated or whole-inactivated vaccine approaches developed by classical methods would not be rapid enough to respond to a pandemic crisis. Therefore, we suggest that the focus for vaccines 


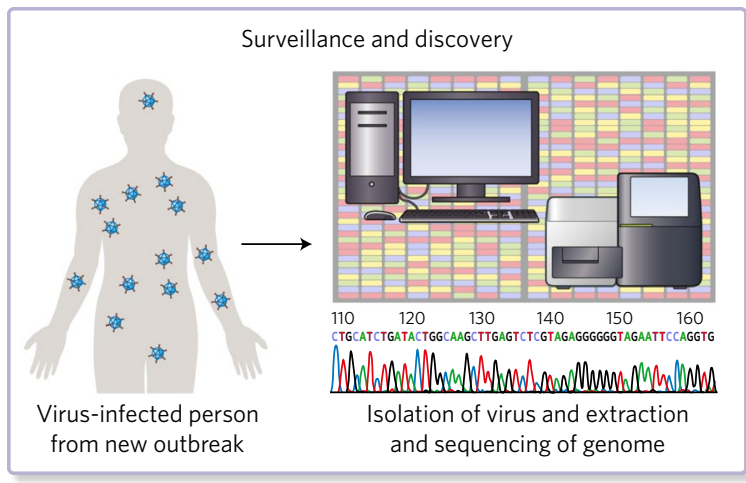

Synthetic vaccinology

Fig. 2 | Synthetic vaccinology. The ability to quickly synthesize nucleic acids has made it possible to rapidly translate sequences identified in the field into reagents needed for the initiation of a vaccine-development process. This can be communicated electronically without the sharing of physical samples, which removes the complexities of shipping and handling biological samples. This is also a practical justification for having surveillance and sequencing ability broadly distributed throughout the world. Credit: Debbie Maizels/Springer Nature.

against EIDs should be based on newer gene-based antigen-delivery technologies, some of which have not yet been licensed for use in humans. Also, in a crisis in which development timelines need to be compressed, using technologies that can provide a candidate vaccine that is based on information derived entirely from target-gene sequences will be more expeditious than procedures that require isolation and growth of a virus that might require high-level containment. This means that even for families of viruses for which there are licensed vaccines, additional approaches beyond liveattenuated and whole-inactivated vaccines should be explored. In parallel, conventional vaccine approaches could be developed as an alternative solution that might be needed in the long term.

Typically, vaccine development is measured in decades. However, since 2009, new technologies have evolved that have provided the tools for reimagining an accelerated process of vaccine development ${ }^{23}$. Key technologies that have changed the pace of vaccine development include the rapid isolation of human monoclonal antibodies ${ }^{24-26}$, the ability to define atomic-level structures of viral surface proteins and how they are organized on viral particles ${ }^{12,27-31}$, next-generation sequencing to characterize both the virus and the repertoire of the host immune response ${ }^{32}$ and methods for defining epitope-specific immune cell phenotypes with more precision at a single-cell level (Fig. 1). Techniques for identifying human monoclonal antibodies by cloning genes encoding paired immunoglobulin heavy and light chains from sorted B cells has been particularly transformative. This method can help define antibody lineages associated with disease progression or immunity and provide precise endpoints for the evaluation of immunogenicity. In addition, monoclonal antibodies are critical reagents not only for assessing the antigenicity of new immunogen designs but also for development as clinical products for passive prophylaxis or therapy.

There are also new concepts that have expedited the process of vaccine discovery. The term 'synthetic vaccinology' is applied when sequencing data alone are sufficient to rapidly initiate vaccine antigen design and the production of key reagents ${ }^{33}$ (Fig. 2). Advances in atomic-level protein engineering ${ }^{10,34,35}$, understanding of the assembly of virus-like particles (VLPs) ${ }^{36-38}$ and the identification of other self-assembling molecules that improve immunogenicity by optimizing antigen display ${ }^{39-41}$ facilitate the fine tuning of vaccineinduced immune responses to specific antigenic targets.

Accelerating the development and evaluation of vaccines will require new investments and international cooperation. Platform technologies that take advantage of delivery approaches with an established manufacturing process or that have a substantial pre-existing safety database in humans could shorten the timeline for manufacture by having uniform 'Chemical, Manufacturing, and Controls' sections for investigational new drug applications and by accelerating preclinical toxicology testing ${ }^{42-51}$ (Fig. 1). This is a major focus of the Coalition for Epidemic Preparedness Innovation and the new concepts of the Biodefense Preparedness Organizations mentioned above, which indicates that the need is being recognized and an infrastructure for investment is being established. It is likely that multiple distinct platform vaccine technologies will be needed to address the diversity of viral pathogens and disease manifestations. In addition to the process of putting product in a vial, there is a need for improved communication and cooperation across borders to accelerate regulatory review of new products. Organizations like the African Vaccine Regulatory Forum should be expanded across the globe so that products can enter efficacy evaluation more rapidly in the midst of a crisis.

\section{Scope of the problem}

Within the 23 families of viruses associated with human infection (Tables 2 and 3), there are multiple genera and species, each of which could be better defined by modern sequencing technology. As the full range of known viral pathogens is defined through sequencing, they could be further categorized according to entry mechanism, tropism or route of transmission to select a finite number of representative viruses to serve as prototypes against which newly emerging viruses can be compared to identify potential vaccine strategies. There might be more than one prototypic virus for each family, and taxonomy is evolving. For example, it is likely that the vaccine approach for the orthomyxovirus influenza virus will be distinct from that for the tick-borne Bourbon virus, which is also an orthomyxovirus, due to differences in transmission route and tropism. Conversely, the Pneumoviridae family has now been split from the Paramyxoviridae family, and while there are subtle differences in fusion-protein structures, genome organization and regulatory proteins, a common vaccine approach might be applicable.

\section{Case studies: reacting to emerging viral diseases}

The 2014 Ebola crisis in West Africa and the 2016 Zika outbreak in South and Central America and the Caribbean revealed several critical features of global health infrastructure and the capacity to respond to infectious disease emergencies. These outbreaks arrived in the context of a 35-year HIV-1 pandemic, as well as multiple instances of newly recognized acute viral diseases, such as chikungunya, 
Table 2 | Families of viruses known to cause human infection

\begin{tabular}{|c|c|c|}
\hline Family & Prototypic virus(es) & Licensed vaccine(s) \\
\hline Paramyxoviridae ${ }^{a}$ & Measles virus, mumps virus, Nipah virus ${ }^{\mathrm{d}, \mathrm{e}}$ & Live-attenuated \\
\hline Togaviridae $^{a}$ & Rubella virus & Live-attenuated \\
\hline Orthomyxoviridae ${ }^{a}$ & Influenza virus $A$ and $B$ & Live-attenuated, whole-inactivated \\
\hline Adenoviridae $^{a}$ & Adenovirus 4 and 7 & Live-attenuated \\
\hline Rhabdoviridae $^{\text {a }}$ & Rabies virus & Live-attenuated \\
\hline Papillomaviridae $^{a}$ & HPV 6, 11,16 and 18 & VLP \\
\hline Poxviridae & Variola virus & Live-attenuated \\
\hline Hepadnaviridae $^{a}$ & Hepatitis B virus & VLP \\
\hline Herpesviridae $^{a}$ & Varicella virus & Live-attenuated \\
\hline Flaviviridae $^{a}$ & Yellow fever virus; TBE; JE; Dengue virus & Live-attenuated, whole-inactivated, live-chimeric \\
\hline Retroviridae $^{b}$ & HIV-1 & \\
\hline Coronaviridae ${ }^{b}$ & SARS $;$ MERS d,e & \\
\hline Parvoviridae ${ }^{b}$ & B19 virus; bocavirus & \\
\hline Caliciviridae $^{b}$ & Norovirus & \\
\hline Polyomaviridae $^{c}$ & $\mathrm{JC}$ virus; $\mathrm{BK}$ virus & \\
\hline Arenaviridae $^{c}$ & Lassa virus ${ }^{\mathrm{d}}$, Machupo virus & \\
\hline Bunyaviridae $^{c}$ & Hantavirus; Rift Valley virus & \\
\hline Astroviridae ${ }^{c}$ & Astrovirus & \\
\hline
\end{tabular}

TBE, tick-borne encephalitis; JE, Japanese encephalitis; RSV, respiratory syncytial virus; MERS, Middle Eastern respiratory syndrome. ${ }^{\mathrm{a} F a m i l i e s}$ with at least one representative licensed vaccine. ${ }^{\mathrm{b}}$ Viruses with active vaccine research. 'Viruses with minimal vaccine research activity. ${ }^{\mathrm{C} V}$ iruses selected by the Coalition for Epidemic Preparedness and Innovation for vaccine-development support. ${ }^{e}$ Viruses of concern listed by the WHO, plus Crimean Congo hemorrhagic fever under Bunyaviruses (Table 3).

in the same regions as Zika. In addition, known diseases are occurring in new populations with distinct epidemiological features, exemplified by the extensive Ebola epidemic in West Africa and MERS, which results from a SARS-like beta-coronavirus that causes sporadic outbreaks of severe respiratory disease, particularly in healthcare settings. While there have been biological, technical and geopolitical hurdles to overcome in emergency responses, each event has driven substantial advances in the overall understanding of viral pathogenesis and immunity. The response to HIV-1, although it was delayed, ushered in the modern era of immunology, and the funding and scientific effort devoted to the biology of HIV-1 and preventing infection with this virus have provided many of the tools needed for other EIDs. Gene-based vaccines, including nucleic-acid or vectored approaches, multi-parameter flow cytometry for better definition of the phenotype and function of cells involved in immunity, multiple techniques for the isolation of neutralizing human monoclonal antibodies, the application of atomic-level structure to antigen design and the definition of B cell clonal lineages as a way of understanding the ontogeny of effective antibody responses all arose as widely used technologies as a consequence of broad and intensive research to combat HIV.

The responses to Ebola and Zika are notable examples of accelerated rapid response, because there was a concerted effort to develop and test experimental vaccine candidates in the field during the outbreak and to use them as an intervention tool. Understanding the conditions under which these responses have been feasible, in addition to awareness of the technological tools currently available, might improve the ability to utilize vaccines in future outbreaks that threaten the public health.
Since its discovery in 1976, Zaire ebolavirus and other related filoviruses (Sudan ebolavirus and Marburgvirus) have caused sporadic outbreaks, each affecting on the order of a few hundred people within a geographically limited area. While those outbreaks were alarming, there was no indication until 2014 that filoviruses are able to spread as a large epidemic through both urban centers and rural areas. After Ebola re-emerged in the 1995 Kikwit epidemic, work on filovirus pathogenesis, immunity and vaccine development was carried out by relatively few investigators because of the need for a biosafety level 4 containment space and limited commercial interest to support advanced development. That changed in 2001, when a greater emphasis was placed on biodefense research as a result of the anthrax attacks on postal facilities and government offices. The US government recognized a gap in preparedness for natural or intentional exposure to highly lethal pathogens, and Operation Bioshield was launched as a mechanism for funding research and development for countermeasures against a list of specific, highly lethal agents (category A pathogens). Research funding was complemented by new rules from the US FDA to facilitate the licensure of vaccines and therapeutics with animal data. Since the efficacy of vaccines directed against category A lethal pathogens cannot be evaluated easily in humans, the FDA Animal Rule (21 CFR 314.601.90) was proposed to provide a pathway for the regulatory review of biological countermeasures against such pathogens on the basis of efficacy studies in animals instead of humans, with the definition of immunological correlates of protection in well-defined animal models that recapitulate the pathogenesis of human disease, and bridging studies in humans for safety and immunogenicity. 


\section{Table 3 | Other viruses of concern}

\begin{tabular}{|c|c|}
\hline Family & Subfamily, strain or serotype \\
\hline Paramyxoviridae & Hendra virus, Cedar virus, PIV1-PIV3 \\
\hline Togaviridae-alphaviridae & $\begin{array}{l}\text { Chikungunya virus, Western equine encephalitis virus, Eastern equine encephalitis virus, Venezuelan equine } \\
\text { encephalitis virus, Mayaro virus, Ross River virus, Barmah Forest virus, O'nyong'nyong virus, Semliki Forest virus, Getah } \\
\text { virus, Sindbis virus }\end{array}$ \\
\hline Reoviridae & New rotaviruses, Banna virus, Nelson Bay orthoreoviruses \\
\hline Orthomyxoviridae & Multiple subtypes of influenza A virus, Dhori virus, Thogoto virus, Bourbon virus \\
\hline Adenoviridae & Adenovirus 14 or 81 or other serotypes \\
\hline Rhabdoviridae & VSV \\
\hline Picornaviridae & EV71, EV-D68, rhinoviruses, Ljungan virus \\
\hline Papillomaviridae & Other HPV serotypes \\
\hline Poxviridae & Monkeypox virus \\
\hline Herpesviridae & CMV, EBV, HSV-1, HSV-2, HHV-6, HHV-7, HHV-8 \\
\hline Flaviviridae & $\begin{array}{l}\text { HCV, Zika virus, St. Louis encephalitis virus, West Nile virus, Powassan virus, Omsk hemorrhagic fever virus, Murray } \\
\text { Valley encephalitis virus, Rocio encephalitis virus, Kyasanur forest virus, Alkhurma virus, Russian spring and summer } \\
\text { encephalitis virus, Central European tick-borne encephalitis virus, Wesselsbron virus, Bussuquara virus, Cacipacore virus, } \\
\text { Ilheus virus, Iguape virus, Usutu virus }\end{array}$ \\
\hline Bunyaviridae & $\begin{array}{l}\text { Crimean Congo hemorrhagic fever virus, California encephalitis virus, Batai virus, Bhanja virus, Dobrava-Belgrade virus, } \\
\text { Erve virus, Puumala virus, Seoul virus, Tahyna virus, severe fever with thrombocytopenia syndrome virus, La Crosse } \\
\text { encephalitis virus, Cache Valley virus, Jamestown Canyon virus, snowshoe hare virus, Heartland virus, Oropouche virus }\end{array}$ \\
\hline Arenaviridae & Junin virus, Guanarito virus, Chapare virus, Sabia virus, Flexal virus, lymphocytic choriomeningitis virus, Lujo virus \\
\hline Polyomaviridae & SV40, Merkel cell virus \\
\hline Arteriviridae ${ }^{a}$ & Simian hemorrhagic fever virus \\
\hline
\end{tabular}

Viruses of concern not included among the prototypic viruses in Table 2. PIV, parainfluenza virus; EV, enterovirus; CMV, cytomegalovirus; EBV, Epstein-Barr virus; HSV, herpes simplex virus; HHV, human herpesvirus; $\mathrm{HCV}$, hepatitis $\mathrm{C}$ virus; $\mathrm{SV}$, simian virus. ${ }^{a}$ Not yet reported to infect humans.

The increased support for identifying interventions for pathogens with potential for use in biowarfare and new regulatory options for clinical development led to the first phase I evaluation of a candidate vaccine against Ebola virus in 2003 ( refs $^{52,53}$ ). A series of subsequent clinical trials of both DNA and recombinant adenovirus vectored vaccines proceeded over the ensuing 10 years and included optimization of vaccine components and refinement of the surface-glycoprotein-antigen design. Extensive studies of vaccine efficacy and correlates of immunity guided the development of these vaccines ${ }^{54-56}$. In parallel, there were substantial advances in understanding the structure, entry mechanisms, replication strategies and pathogenesis of Ebola virus that informed the design of vaccines and clinical trials. Over the course of two decades, this basic research on pathogenesis and immunological correlates of protection, together with clinical evaluation of safety and immunogenicity in phase I trials, formed a critical safety database and foundation of knowledge that allowed the rapid entry of new candidate vaccines against Ebola virus into advanced clinical evaluation during the 2014 outbreak. For example, the final lead adenovirusbased vaccine candidate, ChAd3-EBO, entered phase I evaluation in September 2014, within 1 month of the declaration of a Public Health Emergency of International Concern. With the cooperation and efforts of multiple government agencies, regulatory authorities and funders, a world-wide consortium of sites helped to expand the safety and immunogenicity database and allowed this product to enter efficacy evaluation in Liberia by February 2015 (refs ${ }^{55,57-59}$ ). The established safety data for Ebola virus glycoprotein antigens in humans also facilitated the rapid advance of other glycoproteinexpressing vaccine vectors that had not been tested in humans before, including recombinant vesicular stomatitis virus (rVSV), which was successfully evaluated for efficacy in Guinea in 2015 $\left(\right.$ ref. ${ }^{60}$ ). Despite the efforts for rapid deployment, the ChAd3 vector did not complete efficacy testing in Liberia because there were no more incident cases of Ebola. However, the rVSV vaccine was found to be efficacious in a stepped-wedge ring-vaccination design in Guinea, where the outbreak continued for a few more months. Achieving an efficacy result was possible not only because of prior basic and clinical research but also because the ChAd3-EBO vaccine and rVSV vaccine had been manufactured and vialed by current good manufacturing practice before the 2014 outbreak in West Africa from prior investments by the US government and Canadian government, respectively.

The Ebola experience of a rapid, international response to a global public health emergency illustrates the benefits of preparation in defining the immunological determinants of vaccine protection against a highly lethal pathogen, animal-model development, phase I safety and immunogenicity data, industry involvement and manufactured vaccine available for immediate use. However, it also exposed gaps in the information and planning required for effective use of vaccines to intervene in the midst of a public health crisis.

While the effort to accelerate the availability of a vaccine against Ebola virus for the West African epidemic was extraordinary and unprecedented, it is useful to consider what steps could have been taken in advance make the vaccine available more quickly. Ideally, the vaccine would have already been licensed for general use on the basis of the Animal Rule or would have been pre-authorized for emergency use by regional regulatory authorities and stockpiled in sufficient quantities before the epidemic. Alternatively, unlicensed experimental vaccines prepared for use in humans by current good manufacturing practice could be stockpiled in sufficient quantities and clinical protocols and consent documents could be preapproved by regional regulatory agencies for expanded use. Therefore, if the clinical infrastructure were available, rapid deployment and evaluation of a vaccine could be accomplished. Since achieving licensure through traditional pathways by randomized clinical phase III efficacy trials is unlikely in the absence of ongoing and consistent 
endemic transmission, other provisions for licensure by the Animal Rule or pre-outbreak approvals for clinical efficacy testing during new outbreaks should be proactively established.

Since its discovery in 1947 in the Ziika forest in Uganda, Zika virus was occasionally recognized as a cause of mild dengue-like syndrome with fever and rash ${ }^{61}$. In 2007, it caused an outbreak on Yap island, then in 2013, it emerged in French Polynesia, and the current regional outbreak started in late 2014 in Brazil. The cause of the outbreak was recognized to be Zika virus in May 2015, and the association with fetal infection and neurological disorders was identified in early 2016, when the WHO declared a global health emergency ${ }^{62-64}$.

Unlike the response to Ebola, for Zika, there were no pre-existing basic or clinical research programs and no candidate vaccines available when the outbreak emerged, so having a product authorized for immediate use was not possible. Although Bharat Biotech in Hyderabad, India, had recognized the potential need for a vaccine against Zika virus in 2013 during the French Polynesia outbreak, vaccine development was not accelerated until 2016, in parallel with a variety of platform technologies that advanced rapidly on the basis of the extensive information available from work on other members of the Flaviviridae family ${ }^{65}$. This included a substantial body of knowledge about viral structure ${ }^{6,67}$ and mechanisms of antibody neutralization ${ }^{68-74}$, as well as a precedent for successful vaccine development ${ }^{75-81}$. Vaccines based on nucleic-acid platform technology were available first ${ }^{82-86}$, but vector-based approaches and traditional platforms using whole-inactivated virus, live-attenuated virus or live chimeric viruses were not far behind and were developed in parallel ${ }^{86-90}$. Animal models were rapidly developed ${ }^{91}$, serological assays $^{92}$ and diagnostic tests were established ${ }^{93}$, monoclonal antibodies were isolated ${ }^{24,74,94}$, basic aspects of flavivirus-specific virology were confirmed, including atomic-level structure ${ }^{28,95}$, and large natural-history and epidemiological studies were initiated. Within 7 months of the time of the announcement of the Public Health Emergency of International Concern, phase I clinical trials were initiated, and phase IIb efficacy evaluation of early candidates began within 18 months. Modeling transmission dynamics to anticipate where trial sites should be located, trying to capture the mosquito seasons in both the Southern hemisphere and Northern hemisphere and using infection as well as clinical symptom endpoints might increase the likelihood of achieving an efficacious result. As noted, moving into advanced clinical studies more rapidly would require pre-approved vaccine-delivery platforms and regional efficacy protocols in addition to maintenance of a global clinical trials infrastructure that could be accessed on short notice.

\section{Opportunities for advanced preparation}

In the 21 st century, humans are faced with extraordinary challenges from known and emerging viral pathogens due to increased likelihood of transmission from zoonotic, vector-borne and human sources. Fortunately, the technical ability to identify potential viral threats and understand viral pathogens with atomic level precision is available. Prospective investment will represent a cost-saving measure compared with the expenditures required for emergency responses, but it will require advocacy and sustained political will to support the advanced preparation necessary to prevent or rapidly respond to the next global pandemic.

Surveillance units specialized in high-throughput sequencing should be established in selected locations around the globe where ecosystems are the most diverse. These units could support clinical care by improving regional diagnostic capabilities but could also engage in discovery research by probing samples from patients with fever or rash of unknown origin, samples from domesticated or wild animals in which there is evidence of contagion, urine collected from wild rodents and bats, or the contents of mosquitos and other biting insects, with primers that could capture sequences from the 23 families of viruses that are greatest concern. There might be additional families of viruses, such as Arteriviridae, that cause considerable veterinary disease and present a potential zoonotic risk, since there are known simian viruses within the same order (Nidovirales) ${ }^{96}$.

Within each relevant family of viruses, a database of information with accompanying reagents and assays should be developed for prototypic viruses on the basis of properties of tropism, transmission routes and other distinguishing features of pathogenesis and structure.

Candidate vaccine approaches could be designed on the basis of features of the overall viral structure, transmission dynamics, entry requirements, tropism and replication strategy, and they could be evaluated in small animals for immunogenicity and protection against challenge where feasible.

The step of manufacturing a candidate vaccine for clinical evaluation when there is no immediate market is the most difficult hurdle to overcome from a cost-benefit perspective. Intermediate options could include the manufacture and evaluation of at least one product from each major technology platform through the stages of phase I and II clinical testing, rather than for each family or subgroup of viruses. For example, the steps involved in constructing and manufacturing a product using gene-based antigen delivery by nucleic acids or a replication-defective vector might be the same for any envelope glycoprotein that functions as a trimeric class I fusion 'machine', regardless of the specific virus. Likewise, the steps involved in scaling up the production of a VLP for viruses with icosahedral capsids might be similar. Having a precedent for the manufacture of a particular type of product before the next crisis occurs would improve response time. However, to the extent possible, and because each virus, even within taxonomic families, has unique characteristics, developing rational candidate vaccines directed against at least one prototype virus within each major phenotypic category within each family of viruses is advisable (Table 2). New partnership models to accomplish these tasks using both public funding and private funding might be needed; such models include government-to-government collaboration, nonprofit organizations, philanthropy and public-private partnerships. The experience with Ebola and Zika taught that as media coverage wanes and pressure from the public health crisis diminishes, political will and corporate commitment to long-term vaccine-development efforts can waver.

Knowledge accumulates across pathogens and platforms, so organizing working groups that span viruses with shared features such as entry mechanisms and whether the virus has a lipid envelope or not might be useful. Likewise, establishing groups that can apply particular platform technologies for the design and manufacture of vaccines across multiple pathogens might create synergies. Programs could also be designed around functional categories of viruses or large conceptual gaps in knowledge, as described in the following four examples.

VLPs are a licensed platform for human papillomavirus, hepatitis $B$ virus and hepatitis E virus and are extremely effective immunogens. They are safe for both manufacture and administration. Therefore, it is reasonable to assume that for other non-enveloped viruses that have an icosahedral capsid, especially those with a blood-borne phase as part of their pathogenesis, the virus is likely to be susceptible to neutralizing antibody. An ordered antigen array on a particle is one of the most potent mechanisms for inducing strong antibody responses, and by producing the authentic structures on the native capsid, a VLP approach, using either protein-based delivery or gene-based delivery, would be ideal for these families of viruses.

For enveloped viruses, choosing a platform vaccine approach is not as straightforward, but most have a protein or set of proteins that allow them to mediate membrane fusion. This is often a key target for a neutralizing antibody and therefore these proteins are attractive 
vaccine targets. If the enveloped virus of interest is known to be sensitive to neutralization and the fusion protein (or complex) can be isolated, stabilized and presented in an appropriate conformation, then VLPs, nanoparticles or subunit-protein-vaccine approaches delivered as proteins or as gene-encoded antigens would be rational. Working groups specialized in designing antigens for class I, II or III fusion proteins might be useful within this area of work.

Many enveloped viruses have a complex pathogenesis and might require more than just a neutralizing-antibody response. Ideally, for these types of viruses (such as poxviruses and filoviruses), both neutralizing-antibody responses and cell-mediated responses capable of clearing virus-infected cells and limiting the spread of virus that escapes the front line of antibody defense might be needed for adequate immunity. In this case, gene-based antigen delivery using nucleic acids, single-round replication-defective whole virus or vector-delivered antigens might be needed for primary induction of protective responses, and working groups focused on the induction and regulation of $\mathrm{CD}^{+} \mathrm{T}$ cell immunity would be useful.

Some viruses are particularly adept at avoiding or escaping the immune system. When there are factors such as extreme genetic plasticity and antigenic variability (as with HIV and influenza virus), multiple distinct serotypes (as with rhinoviruses) or persistent infection and latency (as with HIV or herpesviruses), new strategies need to be developed for antigen design, formulation, route and sequence and timing of delivery, and combination of modalities to achieve sufficient breadth and durability to make vaccine development practical. Solving the biological challenges for these 'difficult' viral pathogens might provide solutions that could be used in pre-pandemic vaccine development or rapid vaccine development in response to emerging viral pathogens. We suggest that working groups and scientific consortia be assembled around larger concepts such as how to design vaccine antigens that induce broadly cross-reactive antibodies or vaccine-delivery approaches that establish selected immune effectors in targeted tissue locations.

\section{Conclusion}

Preparation for emerging viral disease will be an inherent component of achieving the UN SDGs and will require contributions from multiple disciplines, including immunology. Despite the impending threat of pandemic disease from emerging viruses, the ability and technological tools are available to achieve a substantial level of advanced preparation before the next major event, and if a systematic effort can be sustained, effective interventions for the majority of future pathogens can be developed.

Received: 11 September 2017; Accepted: 30 October 2017; Published online: 3 December 2017

\section{References}

1. World Health Organization. The Global Burden of Disease 2004. Part 4:Burden of Disease: DALYs (World Health Organization, 2004).

2. World Health Organization. World Health Statistics 2009. Cause-specific Mortality and Morbidity 47-57 (World Health Organization, 2004).

3. United Nations. Resolution adopted by the General Assembly on 25 September 2015. 70/1. Transforming our world: the 2030 Agenda for Sustainable Development http://www.un.org/ga/search/view_doc.asp?symbol= A/RES/70/1\&Lang=E (2015).

4. Fauci, A. S. \& Morens, D. M. The perpetual challenge of infectious diseases. N. Engl. J. Med. 366, 454-461 (2012).

5. Zhou, T. et al. Structural basis for broad and potent neutralization of HIV-1 by antibody VRC01. Science 329, 811-817 (2010)

6. Li, Y. et al. Mechanism of neutralization by the broadly neutralizing HIV-1 monoclonal antibody VRC01. J. Virol. 85, 8954-8967 (2011).

7. Wu, X. et al. Focused evolution of HIV-1 neutralizing antibodies revealed by structures and deep sequencing. Science 333, 1593-1602 (2011).

8. Joyce, M. G. et al. Vaccine-induced antibodies that neutralize group 1 and group 2 influenza a viruses. Cell 166, 609-623 (2016).

9. Villar, R. F. et al. Reconstituted B cell receptor signaling reveals carbohydratedependent mode of activation. Sci. Rep 6, 36298 (2016).
10. Jardine, J. et al. Rational HIV immunogen design to target specific germline B cell receptors. Science 340, 711-716, https://doi.org/10.1126/ science.1234150 (2013).

11. Andrews, S. F., Graham, B. S., Mascola, J. R. \& McDermott, A. B. Is it possible to develop a "universal" influenza virus vaccine? Immunogenetic considerations underlying b-cell biology in the development of a pan-subtype influenza a vaccine targeting the hemagglutinin stem. Cold Spring Harb. Perspect. Biol. https://doi.org/10.1101/cshperspect.a029413 (2017).

12. McLellan, J. S. et al. Structure of RSV fusion glycoprotein trimer bound to a prefusion-specific neutralizing antibody. Science 340, 1113-1117 (2013).

13. Yassine, H. M. et al. Hemagglutinin-stem nanoparticles generate heterosubtypic influenza protection. Nat. Med 21, 1065-1070 (2015).

14. Tian, M. et al. Induction of HIV neutralizing antibody lineages in mice with diverse precursor repertoires. Cell 166, 1471-1484 (2016).

15. Hahn, B. H., Shaw, G. M., De Cock, K. M. \& Sharp, P. M. AIDS as a zoonosis: scientific and public health implications. Science 287, 607-614 (2000).

16. Drosten, C. et al. Identification of a novel coronavirus in patients with severe acute respiratory syndrome. N. Engl. J. Med. 348, 1967-1976 (2003).

17. Peiris, J. S., Yuen, K. Y., Osterhaus, A. D. \& Stöhr, K. The severe acute respiratory syndrome. N. Engl. J. Med. 349, 2431-2441 (2003).

18. Ungchusak, K. et al. Probable person-to-person transmission of avian influenza A (H5N1). N. Engl. J. Med. 352, 333-340 (2005).

19. Centers for Disease Control and Prevention (CDC). Outbreaks of avian influenza A (H5N1) in Asia and interim recommendations for evaluation and reporting of suspected cases-United States, 2004. MMWR Morb. Mortal. Wkly. Rep. 53, 97-100 (2004).

20. Plotkin, S. A., Mahmoud, A. A. \& Farrar, J. Establishing a global vaccinedevelopment fund. N. Engl. J. Med. 373, 297-300 (2015).

21. Plotkin, S. A. Vaccines for epidemic infections and the role of CEPI. Hum. Vaccin. Immunother. http://dx.doi.org/10.1080/21645515.2017.1306615 (2017).

22. Slaoui, M. \& Mullard, A. Moncef Slaoui. Nat. Rev. Drug Discov. 14 452-453 (2015).

23. Graham, B. S. Advances in antiviral vaccine development. Immunol. Rev. 255, 230-242 (2013)

24. Stettler, K. et al. Specificity, cross-reactivity, and function of antibodies elicited by Zika virus infection. Science 353, 823-826 (2016).

25. Wrammert, J. et al. Rapid cloning of high-affinity human monoclonal antibodies against influenza virus. Nature 453, 667-671 (2008).

26. Gilman, M. S. et al. Rapid profiling of RSV antibody repertoires from the memory B cells of naturally infected adult donors. Sci. Immunol. 1, eaaj1879 (2016)

27. McLellan, J. S., Yang, Y., Graham, B. S. \& Kwong, P. D. Structure of respiratory syncytial virus fusion glycoprotein in the postfusion conformation reveals preservation of neutralizing epitopes. J. Virol. 85, 7788-7796 (2011).

28. Sirohi, D. et al. The $3.8 \AA$ resolution cryo-EM structure of Zika virus. Science 352, 467-470 (2016)

29. Wyatt, R. et al. The antigenic structure of the HIV gp120 envelope glycoprotein. Nature 393, 705-711 (1998).

30. Voss, J. E. et al. Glycoprotein organization of Chikungunya virus particles revealed by X-ray crystallography. Nature 468, 709-712 (2010).

31. Wilson, I. A., Skehel, J. J. \& Wiley, D. C. Structure of the haemagglutinin membrane glycoprotein of influenza virus at $3 \mathrm{~A}$ resolution. Nature $\mathbf{2 8 9}$, 366-373 (1981)

32. Furman, D. \& Davis, M. M. New approaches to understanding the immune response to vaccination and infection. Vaccine 33, 5271-5281 (2015).

33. Dormitzer, P. R. Rapid production of synthetic influenza vaccines. Curr. Top. Microbiol. Immunol. 386, 237-273 (2015).

34. Joyce, M. G. et al. Outer domain of HIV-1 gp120: antigenic optimization, structural malleability, and crystal structure with antibody VRC-PG04. J. Virol. 87, 2294-2306 (2013).

35 . $\mathrm{Wu}, \mathrm{X}$. et al. Rational design of envelope identifies broadly neutralizing human monoclonal antibodies to HIV-1. Science 329, 856-861 (2010).

36. Li, L. et al. The flavivirus precursor membrane-envelope protein complex: structure and maturation. Science 319, 1830-1834 (2008).

37. Mukhopadhyay, S., Kuhn, R. J. \& Rossmann, M. G. A structural perspective of the flavivirus life cycle. Nat. Rev. Microbiol. 3, 13-22 (2005).

38. Buck, C. B., Pastrana, D. V., Lowy, D. R. \& Schiller, J. T. Efficient intracellular assembly of papillomaviral vectors. J. Virol. 78, 751-757 (2004).

39. Kanekiyo, M. et al. Self-assembling influenza nanoparticle vaccines elicit broadly neutralizing H1N1 antibodies. Nature 499, 102-106 (2013).

40. King, N. P. et al. Computational design of self-assembling protein nanomaterials with atomic level accuracy. Science 336, 1171-1174 (2012)

41. King, N. P. et al. Accurate design of co-assembling multi-component protein nanomaterials. Nature 510, 103-108 (2014).

42. Gilbert, S. C. \& Warimwe, G. M. Rapid development of vaccines against emerging pathogens: The replication-deficient simian adenovirus platform technology. Vaccine 35, 4461-4464 (2017). 
43. Mühlebach, M. D. Vaccine platform recombinant measles virus. Virus Genes (2017)

44. Volz, A. \& Sutter, G. Modified vaccinia virus ankara: history, value in basic research, and current perspectives for vaccine development. Adv. Virus Res 97, 187-243 (2017).

45. Effio, C. L. \& Hubbuch, J. Next generation vaccines and vectors: Designing downstream processes for recombinant protein-based virus-like particles. Biotechnol. J 10, 715-727 (2015).

46. Suschak, J. J., Williams, J. A. \& Schmaljohn, C. S. Advancements in DNA vaccine vectors, non-mechanical delivery methods, and molecular adjuvants to increase immunogenicity. Hum. Vaccin. Immunother. http://dx.doi.org/10.1 080/21645515.2017.1330236 (2017)

47. Bahl, K. et al. Preclinical and clinical demonstration of immunogenicity by mRNA vaccines against $\mathrm{H} 10 \mathrm{~N} 8$ and $\mathrm{H} 7 \mathrm{~N} 9$ influenza viruses. Mol. Ther. 25, 1316-1327 (2017).

48. Ulmer, J. B. \& Geall, A. J. Recent innovations in mRNA vaccines. Curr. Opin. Immunol. 41, 18-22 (2016).

49. Ledgerwood, J. E. \& Graham, B. S. DNA vaccines: a safe and efficien platform technology for responding to emerging infectious diseases. Hum. Vaccin 5, 623-626 (2009).

50. Didierlaurent, A. M. et al. Adjuvant system AS01: helping to overcome the challenges of modern vaccines. Expert Rev. Vaccines 16, 55-63 (2017).

51. Prausnitz, M. R. Engineering microneedle patches for vaccination and drug delivery to skin. Annu. Rev. Chem. Biomol. Eng. 8, 177-200 (2017).

52. Martin, J. E. et al. A DNA vaccine for Ebola virus is safe and immunogenic in a phase I clinical trial. Clin. Vaccine Immunol. 13, 1267-1277 (2006).

53. Sullivan, N. J., Sanchez, A., Rollin, P. E., Yang, Z. Y. \& Nabel, G. J. Development of a preventive vaccine for Ebola virus infection in primates. Nature 408, 605-609 (2000).

54. Sullivan, N. J. et al. CD8 ${ }^{+}$cellular immunity mediates rAd5 vaccine protection against Ebola virus infection of nonhuman primates. Nat. Med 17, 1128-1131 (2011).

55. Stanley, D. A. et al. Chimpanzee adenovirus vaccine generates acute and durable protective immunity against ebolavirus challenge. Nat. Med 20, 1126-1129 (2014).

56. Zhou, Y. \& Sullivan, N. J. Immunology and evolvement of the adenovirus prime, MVA boost Ebola virus vaccine. Curr. Opin. Immunol. 35, 131-136 (2015).

57. Ledgerwood, J. E. et al. Chimpanzee adenovirus vector Ebola vaccine. N. Engl. J. Med. 376, 928-938 (2017).

58. Ewer, K. et al. A monovalent chimpanzee adenovirus Ebola vaccine boosted with MVA. N. Engl. J. Med. 374, 1635-1646 (2016).

59. De Santis, O. et al. Safety and immunogenicity of a chimpanzee adenovirusvectored Ebola vaccine in healthy adults: a randomised, double-blind, placebo-controlled, dose-finding, phase 1/2a study. Lancet Infect. Dis. 16, 311-320 (2016).

60. Huttner, A. et al. The effect of dose on the safety and immunogenicity of the VSV Ebola candidate vaccine: a randomised double-blind, placebo-controlled phase 1/2 trial. Lancet Infect. Dis. 15, 1156-1166 (2015).

61. Dick, G. W. \& Haddow, A. J. Uganda S virus; a hitherto unrecorded virus isolated from mosquitoes in Uganda. I. Isolation and pathogenicity. Trans. $R$. Soc. Trop. Med. Hyg. 46, 600-618 (1952).

62. Cauchemez, S. et al. Association between Zika virus and microcephaly in French Polynesia, 2013-15: a retrospective study. Lancet 387, 2125-2132 (2016)

63. Brasil, P. et al. Zika virus infection in pregnant women in Rio de Janeiro. N. Engl. J. Med. 375, 2321-2334 (2016).

64. Duffy, M. R. et al. Zika virus outbreak on Yap Island, Federated States of Micronesia. N. Engl. J. Med. 360, 2536-2543 (2009).

65. Pierson, T. C. \& Graham, B. S. Zika virus: immunity and vaccine development. Cell 167, 625-631 (2016)

66. Rey, F. A., Heinz, F. X., Mandl, C., Kunz, C. \& Harrison, S. C. The envelope glycoprotein from tick-borne encephalitis virus at $2 \mathrm{~A}$ resolution. Nature $\mathbf{3 7 5}$, 291-298 (1995).

67. Heinz, F. X. \& Stiasny, K. Flaviviruses and their antigenic structure. J. Clin. Virol. 55, 289-295 (2012).

68. Van Gessel, Y. et al. Correlation of protection against Japanese encephalitis virus and JE vaccine (IXIARO $\left(^{\circ}\right)$ ) induced neutralizing antibody titers. Vaccine 29, 5925-5931 (2011).

69. Julander, J. G., Trent, D. W. \& Monath, T. P. Immune correlates of protection against yellow fever determined by passive immunization and challenge in the hamster model. Vaccine 29, 6008-6016 (2011).

70. Kreil, T. R., Burger, I., Bachmann, M., Fraiss, S. \& Eibl, M. M. Antibodies protect mice against challenge with tick-borne encephalitis virus (TBEV)infected macrophages. Clin. Exp. Immunol. 110, 358-361 (1997).

71. Rouvinski, A. et al. Recognition determinants of broadly neutralizing human antibodies against dengue viruses. Nature 520, 109-113 (2015).
72. Kaufmann, B. et al. Neutralization of West Nile virus by cross-linking of its surface proteins with Fab fragments of the human monoclonal antibody CR4354. Proc. Natl. Acad. Sci. USA 107, 18950-18955 (2010).

73. de Alwis, R. et al. Identification of human neutralizing antibodies that bind to complex epitopes on dengue virions. Proc. Natl. Acad. Sci. USA 109, 7439-7444 (2012)

74. Zhao, H. et al. Structural basis of Zika virus-specific antibody protection. Cell 166, 1016-1027 (2016).

75. Šmit, R. \& Postma, M. J. Review of tick-borne encephalitis and vaccines: clinical and economical aspects. Expert Rev. Vaccines 14, 737-747 (2015).

76. Halstead, S. B. \& Thomas, S. J. New Japanese encephalitis vaccines: alternatives to production in mouse brain. Expert Rev. Vaccines 10, 355-364 (2011).

77. Staples, J. E. \& Monath, T. P. Yellow fever: 100 years of discovery. J. Am. Med. Assoc 300, 960-962 (2008).

78. Guy, B. et al. From research to phase III: preclinical, industrial and clinical development of the Sanofi Pasteur tetravalent dengue vaccine. Vaccine 29, 7229-7241 (2011).

79. Hadinegoro, S. R. et al. Efficacy and long-term safety of a dengue vaccine in regions of endemic disease. N. Engl. J. Med. 373, 1195-1206 (2015).

80. Durbin, A. P., Kirkpatrick, B. D., Pierce, K. K., Schmidt, A. C. \& Whitehead, S. S. Development and clinical evaluation of multiple investigational monovalent DENV vaccines to identify components for inclusion in a live attenuated tetravalent DENV vaccine. Vaccine 29, 7242-7250 (2011).

81. Whitehead, S. S. et al. In a randomized trial, the live attenuated tetravalent dengue vaccine TV003 is well-tolerated and highly immunogenic in subjects with flavivirus exposure prior to vaccination. PLoS Negl. Trop. Dis. 11, e0005584 (2017)

82. Muthumani, K. et al. In vivo protection against ZIKV infection and pathogenesis through passive antibody transfer and active immunisation with a prMEnv DNA vaccine. Npj Vaccines 1, 16021 (2016).

83. Dowd, K. A. et al. Rapid development of a DNA vaccine for Zika virus Science 354, 237-240

84. Pardi, N. et al. Zika virus protection by a single low-dose nucleosidemodified mRNA vaccination. Nature 543, 248-251 (2017).

85. Richner, J. M. et al. Modified mRNA vaccines protect against Zika virus infection. Cell 169, 176 (2017).

86. Tauber, E. et al. Safety and immunogenicity of a Vero-cell-derived, inactivated Japanese encephalitis vaccine: a non-inferiority, phase III, randomised controlled trial. Lancet 370, 1847-1853 (2007).

87. Shan, C. et al. A live-attenuated Zika virus vaccine candidate induces sterilizing immunity in mouse models. Nat. Med 23, 763-767 (2017).

88. Sumathy, K. et al. Protective efficacy of Zika vaccine in AG129 mouse model. Sci. Rep 7, 46375 (2017).

89. Abbink, P. et al. Protective efficacy of multiple vaccine platforms against Zika virus challenge in rhesus monkeys. Science 353, 1129-1132 (2016).

90. Larocca, R. A. et al. Vaccine protection against Zika virus from Brazil. Nature 536, 474-478 (2016)

91. Morrison, T. E. \& Diamond, M. S. Animal models of Zika virus infection, pathogenesis, and immunity. J. Virol. 91, e00009-17 (2017).

92. Dowd, K. A. et al. Broadly neutralizing activity of Zika virus-immune sera identifies a single viral serotype. Cell Reports 16, 1485-1491 (2016).

93. US Food \& Drug Administration. Emergency Use Authorizations. https://www.fda.gov/medicaldevices/safety/emergencysituations/ucm 161496 htm (2016).

94. Sapparapu, G. et al. Neutralizing human antibodies prevent Zika virus replication and fetal disease in mice. Nature 540, 443-447 (2016).

95. Grant, A. et al. Zika virus targets human STAT2 to inhibit type I interferon signaling. Cell Host Microbe 19, 882-890 (2016).

96. Bailey, A. L. et al. Zoonotic potential of simian arteriviruses. J. Virol. 90, 630-635 (2015)

\section{Acknowledgements}

We thank J. Mascola, K. Kelley, T. Pierson and K. Morabito for editorial comments, and A. Fauci, H. Achincloss, J. Gall, K. Modjarrad, S. Plotkin and C. Cassetti for discussions. Supported by the National Institute of Allergy and Infectious Diseases (intramural funding).

\section{Competing interests}

The authors declare no competing financial interests.

\section{Additional information}

Reprints and permissions information is available at www.nature.com/reprints. Correspondence and requests for materials should be addressed to B.S.G. or N.J.S.

Publisher's note: Springer Nature remains neutral with regard to jurisdictional claims in published maps and institutional affiliations. 